\title{
Risk of statin therapy in elderly
}

\author{
Ginter $\mathrm{E}^{1}$, Kajaba $\mathrm{I}^{2}$ \\ Slovak Medical University, Bratislava, Slovakia. ginter.emil@mail.t-com.sk
}

Text in PDF www.elis.sk.

A meta-analysis of younger patients included in randomised trials found good evidence that statins reduce vascular events and mortality in people with high cholesterol and the risk of coronary heart disease (1). The use of statin in the elderly prevents the disease but can exert considerable side effects. In older adults, an exposure to higher doses of statins increases the risk of adverse effects. From the biochemical aspect, there are at least four factors leading to metabolic disorders: extremely low cholesterol levels, decrease in coenzyme Q10, lowering Ras proteins and decrease in dolichol concentration (Fig. 1).

\section{Extremely low cholesterol levels}

Based on a meta-analysis of 19 studies, NIH conference concluded that men and, to a lesser extent, women with a total serum cholesterol level below $4.2 \mathrm{mmol} / \mathrm{L}$ exhibited about a $10 \%$ to 20 $\%$ excess in total mortality compared with those with a cholesterol level between 4.2 and $5.2 \mathrm{mmol} / \mathrm{L}$. Specifically, the causes of the excess in mortality included cancer (primarily in the lung and haematopoietic tissue), respiratory and digestive disease, violent death (suicide and trauma), and haemorrhagic stroke (2). A great Japanese study (6-year, nationwide cohort study based on 47,294 patients) (3) found extremely high mortality in patients treated with statin with total cholesterol levels under $4.1 \mathrm{mmol} / \mathrm{l}$ (Fig. 2).

Statins inhibite synthesis not only of cholesterol

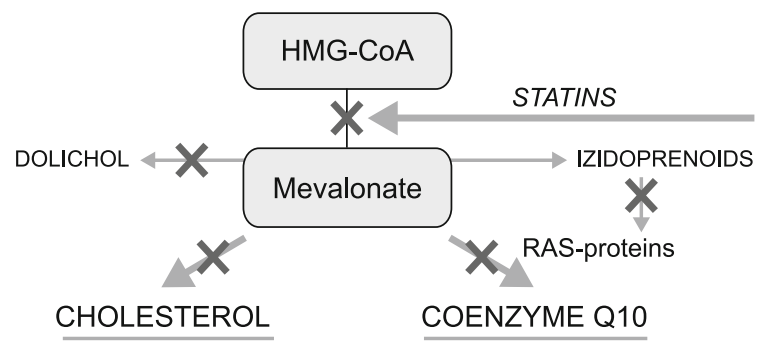

Fig. 1. Statins inhibition have effects not only on cholesterol synthesis

${ }^{1}$ Slovak Medical University, Bratislava, Slovakia, and ${ }^{2}$ CarnoMed, Medical centre, Bratislava, Slovakia

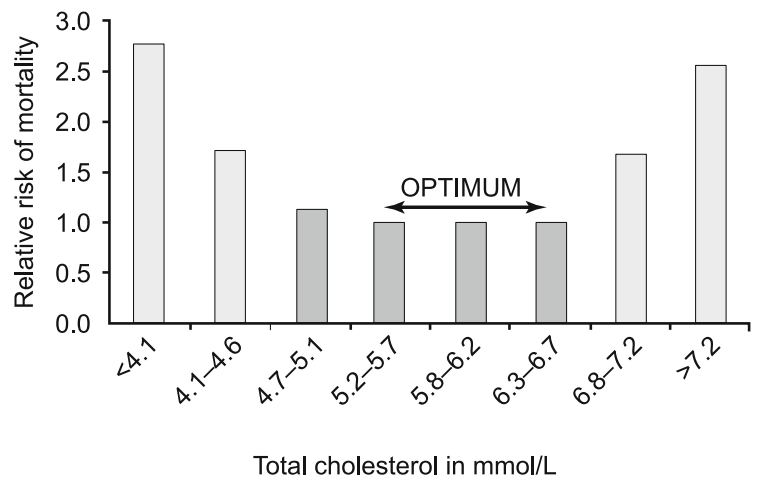

Fig. 2. Very low cholesterol levels increase the risk of total mortality.

The J-curve association was observed also between LDL-C concentrations and total mortality. Malignancy was the most prevalent cause of death.

\section{Decrease in coenzyme Q10}

Coenzyme Q10 also known as ubiquinone is present in most eukaryotic cells, primarily in the mitochondria. It is a component of the electron transport chain and participates in aerobic cellular respiration, which generates energy in the form of ATP. Statins block the production of farnesyl pyrophosphate, an intermediate product in the synthesis of coenzyme Q10. This fact, plus the role of CoQ10 in mitochondrial energy production, has prompted the hypothesis that statin-induced CoQ10 deficiency is involved in the pathogenesis of statin myopathy (4). CoQ10 has been routinely used to treat muscle breakdown developed as a side effect of the use of statin medications. About 10 to 15 percent of people taking statins report myalgia or minor muscle aches and weakness. A smaller number have stronger persistent pain called myopathy associated with rhabdomyolysis (serious degenerative muscle tissue condition).

\section{Lowering Ras proteins}

Ras proteins function as binary molecular switches that control intracellular signalling. These signal pathways control such pro- 


\section{5-556}

cesses as actin cytoskeletal integrity, proliferation, differentiation, cell adhesion, apoptosis, and cell migration. Ras and Ras-related proteins are often deregulated in cancers, leading to increased invasion and metastasis, and decreased apoptosis. Mutations of the three Ras genes convert these genes into active oncogenes. There is some evidence that environmental agents may be involved in the induction of mutations (5).

\section{Decrease in dolichol concentration}

Dolichols play a role in the co-translational modification of proteins known as $\mathrm{N}$-glycosylation in the form of dolichol phosphate. Dolichols function as a membrane anchor for the formation of oligosaccharides (6). Dolichols are the major lipid component of human substantia nigra, i.e. neuromelanin. The concentration of neuromelanin in humans is found to be higher than in other primates. Neuromelanin-containing neurons in substantia nigra undergo neurodegeneration during Parkinson's disease. Neuromelanin concentration increases with age, suggesting a role in neuroprotection. Decrease in dolichols may lead to memory loss. Lower serum cholesterol level may be a state marker of Alzheimer disease (7). Lower serum cholesterol level was associated with worse cognitive function in the community sample.

\section{Conclusion}

Statin use in people over 65 years of age is high. Increasing age is a risk factor for adverse events with statins. Applying the evidence for statins to older individuals therefore requires frequent review and consideration of the therapeutic goals and potential benefits and harms. Statins may be with drawn in frail older people who experience adverse effects from their use. For example, myopathy associated with the use of statins is likely to impact the frail older people with limited musculoskeletal strength.

\section{References}

1. Ginter E, Simko V. Statins: the drugs for the 21st century? Bratisl Lek Listy 2009; 110 (10): 664-668.

2. Meilahn EN. Low serum cholesterol, hazardous to health? Circulation 1995; 92: 2365-2366.

3. Matsuzaki M, Kita T, Mabuchi H et al. Large scale cohort study of the relationship between serum cholesterol concentration and coronary events with low-dose simvastatin therapy in Japanese patients with hypercholesterolemia. Circulat J 2002; 66 (12): 1087-1095.

4. Marcoff L, Thompson PD. The role of coenzyme Q10 in statin-associated myopathy: A Systematic Review. J Am Coll Cardiol 2007; 49 (23): 2231-2237.

5. Bos JL. Ras oncogenes in human cancer: A review. Cancer Res 1989; 49: 4682-4689.

6. Fedorov H, Pickford R, Hook J M et al. Dolichol is the major lipid component of human substantia nigra neuromelanin. J Neurochem 2005; 92 (4): 990-995.

7. Kim JM, Stewart R, Shin IS, Yoon JS. Low cholesterol, cognitive function and Alzheimer s disease in a community population with cognitive impairment. Nutr Health Aging 2002; 6 (5): 320-323. 